\title{
COVERING A GROUP WITH ISOLATORS OF FINITELY MANY SUBGROUPS
}

by PATRIZIA LONGOBARDI, MERCEDE MAJ and AKBAR H. RHEMTULLA

(Received 28 January 1992)

Dedicated to Professor B. H. Neumann for his 80 th birthday

1. Introduction. In [6] B. H. Neumann proved the following beautiful result: if a

group $G$ is covered by finitely many cosets, say $G=\bigcup_{i=1}^{n} x_{i} H_{i}$, then we can omit from the union any $x_{i} H_{i}$ for which $\left|G: H_{i}\right|$ is infinite. In particular, $\left|G: H_{j}\right|$ is finite, for some $j \in\{1, \ldots, n\}$.

In an unpublished result $\mathrm{R}$. Baer characterized the groups covered by finitely many abelian subgroups, they are exactly the centre-by-finite groups [8]. Coverings by nilpotent subgroups or by Engel subgroups and by normal subgroups have been studied, for example, by R. Baer (see [8]), L. C. Kappe [2,1], M. A. Brodie and R. F. Chamberlain [1], and recently by M. J. Tomkinson [9].

In this paper we study groups covered by finitely many isolators of subgroups.

If $H$ is a subgroup of the group $G$, the isolator of $H$ in $G$ is, by definition, the subset

$$
I_{G}(H)=\left\{x \in G \mid x^{n} \in H \text { for some } n>0\right\} \text {. }
$$

We denote by $\mathfrak{X}$ the class of groups $G$ such that, whenever $G=\bigcup_{i=1}^{n} I_{G}\left(H_{i}\right)$, then $G=I_{G}\left(H_{j}\right)$ for some $j \in\{1, \ldots, n\}$.

We prove the following results:

THEOREM A. Let $A$ be a normal abelian subgroup of $G$. If $G / A \in \mathfrak{X}$, then $G \in \mathfrak{X}$. If $G$ is locally soluble, then $G \in \mathfrak{X}$.

From Theorem A, using a result of J. C. Lennox [4], it follows that if $G$ is a finitely generated soluble group and $G=\bigcup_{i=1}^{n} I_{G}\left(H_{i}\right)$, then $\left|G: H_{j}\right|$ is finite, for some $j \epsilon$ $\{1, \ldots, n\}$.

THEOREM B. Let $G=\bigcup_{i=1}^{n} I_{G}\left(H_{i}\right)$, where $H_{1}, \ldots, H_{n}$ are abelian subgroups of $G$.

Then $G=I_{G}\left(H_{j}\right)$ for some $j \in\{1, \ldots, n\}$.

The same conclusion of Theorem B holds if $G=\bigcup_{i=1}^{n} I_{G}\left(H_{i}\right)$, with $H_{1}, \ldots, H_{n}$ subnormal subgroups of $G$ (Theorem C and Corollary 3.2).

Most of the standard notation used comes from [8].

We say that a group $G$ has the isolator property ( $G$ has I.P.) if the isolator of every subgroup of $G$ is itself a subgroup of $G$.

A subgroup $H$ is called isolated if $I_{G}(H)=H$.

Finally, if $H, K$ are subgroups of $G$, then we write $H \sim K$ to mean $I_{G}(H)=I_{G}(K)$.

2. Proof of Theorem A. We begin with some preliminary results:

LEMMA 2.1. If every two generator subgroup of $G$ is in $\mathfrak{X}$, then so is $G$.

Glasgow Math. J. 35 (1993) 253-259. 
Proof. Suppose false, and let $G=\bigcup_{i=1}^{n} I_{G}\left(H_{i}\right)$, where $n \geq 2$ is minimal subject to $G \neq I_{G}\left(H_{i}\right)$ for any $i \in\{1, \ldots, n\}$. By minimality of $n$, there exists $h_{1} \in H_{1}-\left(\bigcup_{i=2}^{n} I_{G}\left(H_{i}\right)\right)$. Similarly there exists $h_{2} \in H_{2}$ such that $h_{2} \in I_{G}\left(H_{1}\right) \cup I_{G}\left(H_{3}\right) \cup \cdots \cup I_{G}\left(H_{n}\right)$. Let $J=$ $\left\langle h_{1}, h_{2}\right\rangle$. Then $J=\bigcup_{i=1}^{n} I_{i}\left(H_{i} \cap J\right)$, and by the hypothesis $J=I_{J}\left(H_{i} \cap J\right)$ for some $i$.

Hence $J \subseteq I_{G}\left(H_{i}\right)$ for some $i$, a contradiction.

LeMma 2.2. $\mathfrak{X}=Q \mathfrak{X}$.

Proof. Easily verified.

Lemma 2.3. Let $H \leq G$ be such that $G=I_{G}(H)$. Then $G \in \mathfrak{X}$ if and only if $H \in \mathfrak{X}$. some $i$.

Proof. Assume $G \in \mathfrak{X}$. If $H=\bigcup_{i=1}^{n} I_{H}\left(K_{i}\right)$, then $G=\bigcup_{i=1}^{n} I_{G}\left(K_{i}\right)$, so that $I_{H}\left(K_{i}\right)=H$ for

Conversely, let $H \in \mathfrak{X}$ and suppose $G=\bigcup_{i=1}^{n} I_{G}\left(H_{i}\right)$. Then $H=\bigcup_{i=1}^{n} I_{H}\left(H \cap H_{i}\right)$ and $H=I_{H}\left(H \cap H_{i}\right)$ for some $i$. Hence $G=I_{G}\left(H_{i}\right)$ for some $i$.

We prove now a weaker version of Theorem $A$.

Lemma 2.4. Let $G=\left\langle a_{1}, \ldots, a_{m}, h\right\rangle$, where $A=\left\langle a_{1}, \ldots, a_{m}\right\rangle^{G}$ is abelian. Then $G \in \mathfrak{X}$.

Proof. If $G / A$ is finite, the result follows easily from 2.3 .

Assume $G / A \cong\langle h\rangle$ infinite. We prove, by induction on $n$, that if $G=I_{G}\left(H_{1}\right) \cup$ $I_{G}\left(H_{2}\right) \cup \ldots \cup I_{G}\left(H_{n}\right) \cup A$, then $G=I_{G}\left(H_{j}\right)$, for some $j \in\{1, \ldots, n\}$. Obviously we can assume $H_{i} A>A$, for every $i$, and so $\left|G: H_{i} A\right|$ is finite. Without loss of generality, we may assume $G=H_{1} A=H_{2} A=\ldots=H_{n} A$. Then $H_{i} \cap A \triangleleft G$, for every $i \in\{1, \ldots, n\}$.

We show that $G /\left(A \cap H_{1} \cap \ldots \cap H_{n}\right)$ is polycyclic; then $G /\left(A \cap H_{1} \cap \ldots \cap H_{n}\right)$ is almost I. P. by a result of Rhemtulla and Wehrfritz [7], and $G \in \mathfrak{X}$.

By a theorem of Lennox and Wiegold [5, Theorem $\mathrm{B}$ ], it suffices to prove that $\left(\langle a, h\rangle\left(A \cap H_{1} \cap \ldots \cap H_{n}\right)\right) /\left(A \cap H_{1} \cap \ldots \cap H_{n}\right)$ is polycyclic for every $a \in A$. Hence, without loss of generality, we can assume $A=\langle a\rangle^{G}$.

First, we show that $G /\left(H_{j} \cap A\right)$ is polycyclic, for some $j \in\{1, \ldots, n\}$.

For every $i \in \mathbb{N}$ there exists $\alpha \in \mathbb{N}$ such that $\left(a h^{i}\right)^{\alpha} \in H_{1} \cup H_{2} \cup \ldots \cup H_{n}$. Then there are $i, s \in \mathbb{N}, s>1$, such that $h^{i} a \in I_{G}\left(H_{j}\right), h^{i s} a \in I_{G}\left(H_{j}\right)$ for the same $j \in\{1, \ldots, n\}$. Hence, for a suitable $\beta \in \mathbb{N}, \quad\left(h^{i} a\right)^{\beta s}=h^{i \beta s} a^{h^{i(f s-1)}} \ldots \ldots a^{h^{i}} a \in H_{j}$ and $\left(h^{i s} a\right)^{\beta}=$ $h^{i s \beta} a^{h^{i s(\beta-1)}} \ldots a^{h^{i s}} a \in H_{j}$, from which $a^{-1} a^{-h^{i}} \ldots a^{-h^{i(\beta s-1)}} a^{h^{i s(\beta-1)}} \ldots a^{h^{i s}} a \in A \cap H_{j}$. But $s>$ 1 , and so $i(\beta s-1)>i s(\beta-1)$. Therefore we have $a^{h^{i(p s-1)}} a^{h^{\alpha_{1}}} \ldots a^{h^{a_{1}}} a^{h^{i}} \in H_{j} \cap A$, with $\alpha_{l}$ suitable integers, $i<\alpha_{l}<i(\beta s-1)$, from which $a^{h^{i\left(f_{s-2}\right)}} \ldots a^{h^{\alpha_{t}-i}} a \in H_{j} \cap A$ and $a^{f(h)} \in H_{j} \cap$ $A$, where $f(h)$ is a polynomial over $\mathbb{Z}$ with leading coefficient and constant term equal to 1. Therefore $G /\left(A \cap H_{j}\right)$ is polycyclic [3]. Assume $j=1$; then $G /\left(A \cap H_{1}\right)$ is polycyclic.

If $n=1$, the result follows. Assume $n>1$. Let $1 \leq l \leq n$ be maximum such that $G /\left(A \cap H_{1} \cap \ldots \cap H_{l}\right)$ is polycyclic. Assume for a contradiction $l<n$. Write $B=A \cap$ $H_{1} \cap \ldots \cap H_{l}$ and let $g \in G-\left(A \cup I_{G}\left(H_{1}\right) \cup \ldots \cup I_{G}\left(H_{n-1}\right)\right)$. Thus $g=c h^{s}$, for some 
$c \in A, s \in \mathbb{Z}, s \neq 0$. Put $K=B\langle g\rangle$, then from $B \leq H_{i}$ it follows $K \cap I_{G}\left(H_{i}\right)=B$ for every $1 \leq i \leq l$, and $K=B \cup I_{K}\left(H_{i+1} \cap K\right) \cup \ldots \cup I_{K}\left(H_{n} \cap K\right)$. Notice that $B$ is finitely generated as a $K$-group. By induction, $K=I_{K}\left(H_{i} \cap K\right)$ for some $j \leq n$, and $K=I_{K}\left(H_{n} \cap K\right)$ since $g \notin A \cup I_{G}\left(H_{1}\right) \ldots \cup I_{G}\left(H_{n-1}\right)$. Arguing as before we get $\left(\langle b, g\rangle\left(B \cap H_{n}\right)\right) /(B \cap$ $\left.H_{n}\right)$ polycyclic for every $b \in B$, and then $\left(\langle b, d h\rangle\left(B \cap H_{n}\right)\right) /\left(B \cap H_{n}\right)$ is polycyclic for every $d \in A$. Hence $\left(\langle b, x\rangle\left(B \cap H_{n}\right)\right) /\left(B \cap H_{n}\right)$ is polycyclic for every $b \in B, x \in G$ and $G /\left(B \cap H_{n}\right)$ is polycyclic by a theorem of Lennox and Wiegold [5, Theorem B], contradicting the maximality of $l$.

Now we can prove Theorem A.

Proof of Theorem $A$. Suppose $A \leq G, A$ abelian, $G / A \in \mathfrak{X}$, and for a contradiction $G \notin \mathfrak{X}$.

Let $n$ be the least integer $>1$ such that $G=\bigcup_{i=1}^{n} I_{G}\left(H_{i}\right), H_{i} \leq G$, but $G \neq I_{G}\left(H_{i}\right)$ for any $i \in\{1, \ldots, n\}$.

First remark that we may assume

(I) $G=\bigcup_{i=1}^{n} I_{G}\left(H_{i}\right), G \neq I_{G}\left(H_{i}\right)$ for any $i \in\{1, \ldots, n\}, G=A H_{1}=\ldots=A H_{l}, A \leq$ $\bigcap_{i=1}^{n-l} H_{l+1}$, where $1 \leq l \leq n$. Moreover $A \cap H_{i} \triangleleft G$, for any $i$.

For, if $I_{G}\left(A H_{1}\right) \neq G$, then replace $H_{1}$ by $A H_{1}$; if $I_{G}\left(A H_{1}\right)=G$, then replace $G$ by $A H_{1}$ and for $i \neq 1$, replace $H_{i}$ by $A H_{1} \cap H_{i}$. Observe that $\bigcup_{i=1}^{n} I_{A H_{1}}\left(A H_{1} \cap H_{i}\right)=A H_{1} \cap$ $\bigcup_{i=1}^{n} I_{G}\left(H_{i}\right)=A H_{1}$, and, by our minimal choice of $n, I_{A H_{1}}\left(A H_{1} \cap H_{i}\right) \neq A H_{1}$ for any $i$. Furthermore the given normal abelian subgroup $A$ is still contained in the new $G, H_{1} \geq A$ or $A H_{1}=G$, and, in both cases. $A \cap H_{1} \triangleleft G$. There exists $i \in\{1, \ldots, n\}$ such that $I_{G}\left(A H_{i}\right)=G$, because $G / A \in \mathfrak{X}$. We may assume $i=1$ and $G=A H_{1}$.

Now suppose we have made the adjustment for the first $r$ subgroups $H_{1}, \ldots, H_{r}$ and for the group $G$ such that:

(*) $A$ is contained in the new $G$, either $H_{i} \geq A$ or $A H_{i}=G$, for any $1 \leq i \leq r$.

Remark that then $A \cap H_{i} \triangleleft G$, for any $1 \leq i \leq r$.

If $I_{G}\left(A H_{r+1}\right) \neq G$, then replace $H_{r+1}$ by $A H_{r+1}$ and observe that $(*)$ is satisfied for $H_{r+1}$ as well. If $I_{G}\left(A H_{r+1}\right)=G$, then replace $G$ by $G_{1}=A H_{r+1}$ and $H_{i}$ by $H_{i} \cap A H_{r+1}$ for all $i$. If $i=r+1$, then $H_{r+1}$ satisfies (*); if $i \leq r$ and $A H_{i}=G$, then $A H_{i} \cap A H_{r+1}=$ $A\left(H_{i} \cap A H_{r+1}\right)=G$; if $i \leq r$ and $A \leq H_{i}$, then $A \leq H_{i} \cap A H_{r+1}$. Hence (*) holds for $H_{i}$, for any $i \leq r+1$.

Thus we have made the adjustment for the first $r+1$ subgroups $H_{1}, \ldots, H_{r+1}$ to satisfy $(*)$. Continue this process until $r=n$. As a result of the above adjustment we may assume (I).

Write $M=A \cap \bigcap_{i=1}^{n} H_{i}$.

Passing, if necessary, to the quotient group $G / M$, we have, without loss of 
generality,

(II) $A \cap \bigcap_{i=1}^{n} H_{i}=1$.

The next step is to show that

(III) $A$ is periodic.

If not, then let $\langle a\rangle$ be infinite, $a \in A$. By (II), $\langle a\rangle \cap H_{i}=1$ for some $i$, say $i=1$. Also, by minimality of $n$, there exists $h \in H_{1}$ such that $h \notin \bigcup_{i=2}^{n} I_{G}\left(H_{i}\right)$. Let $H=\langle a, h\rangle$. Clearly $H=\bigcup_{i=1}^{n} I_{H}\left(H_{i} \cap H\right)$ and $H \neq I_{H}\left(H_{i} \cap H\right)$ for any $i$. But, by Lemma $2.4, H \in \mathfrak{X}$, a contradiction.

Now, let $T$ be a subset of $\{1, \ldots, n\}$ of largest cardinality such that $A K \sim G$, where $K=\bigcap_{i \in T} H_{i}$. For any $j \notin T$, let $K_{j}=K \cap H_{j}$. By (I), $|T| \geq 1$. Pick any $a \in A$.

For each $g \in K-\bigcup_{i \notin T} I_{G}\left(K_{i}\right)$ some power $g^{m}$ of $g$ centralizes $a$ modulo $H_{j} \cap A$ for some $j \in T$. For, if $\left|\left(\left\langle a^{\langle g\rangle}\right\rangle\left(H_{j} \cap A\right)\right) /\left(H_{j} \cap A\right)\right|=\infty$, then $a g^{r} \notin I_{G}\left(H_{j}\right)$ for any non-zero integer $r$. If this happens for all $j \in T$, then $a g^{r}, a g^{s} \in I_{G}\left(H_{i}\right)$ for some $i \notin T, r, s \in \mathbb{N}, r \neq s$. From this we get a contradiction to $g \notin \bigcup_{i \notin T} I_{G}\left(K_{i}\right)$.

Let $C_{j}=\left\langle g \in K \mid[a, g] \in H_{j}\right\rangle$. Then $K \sim \bigcup_{i \notin T} K_{i} \cup \bigcup_{j \in T} C_{j}$, and $K \cap A \leq C_{j}$ for all $j \in T$. Since $A K \sim G, A K / A \sim G / A \in \mathfrak{X}$ and so $K /(K \cap A) \simeq A K / A \in \mathfrak{X}$. Hence either $(A \cap$

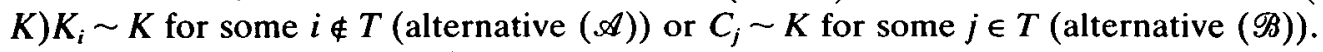

If $(\mathscr{A})$ holds, then $A(A \cap K) K_{i} \sim A K \sim G$, so that $A K_{i} \sim G$, contradicting the maximality of the set $T$.

So assume ( $\mathscr{B})$. For each $a \in A$, let $T_{a}$ be the subset of $T$ such that $C_{i}=C_{i}(a) \sim K$ for all $i \in T_{a}$. Then $T_{a} \neq \varnothing$. For each $j \in T$, let $E_{j}=\left\{a \in A\right.$ such that $\left.j \notin T_{a}\right\}$. Observe that if $a$, $b \in E_{j}$, then $a b \in E_{j}$, for $T_{a b} \supseteq T_{a} \cap T_{b}$. Also $a \in E_{j}$ if and only if $a^{-1} \in E_{j}$. Thus $E_{j} \leq A$, and $A=\bigcup_{j \in T} E_{j}$. Furthermore $E_{j} \triangleleft G$, for any $j \in T$. By B. H. Neumann's result $\left|A: E_{j}\right| \leq|T|$, for some $j \in T$, say $\left|A: E_{1}\right| \leq|T|$ (and $1 \in T$ ). Then for any $g \in K, a \in A$, we have $\left[a, g^{s}\right] \in E_{1}$, for some $s>0$, and, for a suitable $r>0,\left[a, g^{r}, g^{r}\right] \in H_{1} \cap A$ : thus, if $|a|=k$, then $\left[a, g^{r k}\right] \in H_{1} \cap A$. Therefore $E_{1}=A$, so that for any $a \in A$, any $g \in K$, $g^{r} \in C_{1}(a)$ for some $r>0$, and hence $\left[g^{r}, a\right] \in H_{1} \cap A$, so that some suitable power of $a g$ lies in $H_{1}$. This gives $A K \subseteq I_{G}\left(H_{1}\right)$ and $G=I_{G}\left(H_{1}\right)$, a contradiction.

Then $G \in \mathfrak{X}$.

Now assume $G$ locally soluble, we prove that $G \in \mathfrak{X}$. By Lemma 2.1 it suffices to show that every 2-generator subgroup of $G$ is in $\mathfrak{X}$. Thus, without loss of generality, we can assume $G$ soluble, and the result follows easily by induction on the derived length.

COROLlary 2.5. Let $G$ be a finitely generated soluble group.

If $G=I_{G}\left(H_{1}\right) \cup I_{G}\left(H_{2}\right) \cup \ldots \cup I_{G}\left(H_{n}\right)$, with $H_{1}, H_{2}, \ldots, H_{n}$ subgroups of $G$, then $\left|G: H_{i}\right|$ is finite for some $i \in\{1, \ldots, n\}$. 
Proof. We have $G=I_{G}\left(H_{i}\right)$, for some $i \in\{1, \ldots, n\}$, and, by a result of J. Lennox $[4],\left|G: H_{i}\right|$ is finite.

\section{Groups covered by isolators of finitely many abelian subgroups.}

Proof of Theorem B. We argue by induction on $n$. Obviously the result is true for $n=1 ;$ assume $n>1$, and, for a contradiction, $I_{G}\left(H_{i}\right) \subseteq \bigcup_{j \neq i} I_{G}\left(H_{j}\right)$, for any $i$.

First we show that we may assume

(I) $H_{i} \cap H_{j}=1$, for $i \neq j$.

For, if $T \leq G$ and $T \nsubseteq I_{G}\left(H_{i}\right)$ for any $i$, then for every $(h, k), h \neq k, T \cap$ $\left\langle H_{h}, H_{k}\right\rangle \subseteq I_{G}\left(H_{i}\right)$ for any $i$. In fact, if $T \cap\left\langle H_{h}, H_{k}\right\rangle \subseteq I_{G}\left(H_{i}\right)$ for some $i$, then $T \cap H_{h}$, $T \cap H_{k} \subseteq I_{G}\left(H_{i}\right)$ with either $i \neq h$ or $i \neq k$. Assume for example $i \neq h$. Then $T=$ $\bigcup_{j \neq h} I_{T}\left(T \cap H_{j}\right)$ and, by induction, $T=I_{T}\left(T \cap H_{s}\right) \subseteq I_{G}\left(H_{s}\right)$ for some $s$, a contradiction.

Now write $X=\bigcap_{1 \leq i \neq j \leq n}\left\langle H_{i}, H_{j}\right\rangle$. Then it is easy to see that $X \nsubseteq I_{X}\left(H_{i} \cap X\right)$ for any $i$, and we can assume $G=X$, so that $H_{i} \cap H_{j} \triangleleft G$ for any $i \neq j$. Put $Y=\prod_{1 \leq i \neq j \leq n}\left(H_{i} \cap H_{j}\right)$. Then $Y \triangleleft G$ and $Y$ is soluble. If $G / Y \subseteq I_{G / Y}\left(H_{j} Y / Y\right)$ for some $j \in\{1, \ldots, n\}$, then $G \sim H_{j} Y$. But $H_{j} Y$ is soluble, thus, by Theorem $\mathrm{A}, H_{j} Y \sim H_{s} \cap H_{j} Y$ for some $s \in$ $\{1, \ldots, n\}$ and $G \sim H_{s}$, a contradiction. Then we can assume $Y=1$ and (I) holds.

Now we prove that

(II) for every $i \in\{1, \ldots, n\}$ and for every $g \in G$, there exists $\alpha=\alpha(i, g) \in \mathbb{N}$ such that $\left\langle H_{i}, H_{i}^{g^{\alpha}}\right\rangle \subseteq I_{G}\left(H_{i}\right)$.

Let $a \in H_{i}-\left(\bigcup_{j \neq i} I_{G}\left(H_{j}\right)\right)$. Then, for some $h, k, h<k, a^{g^{h}}$ and $a^{g^{k}}$ are in $I_{G}\left(H_{s}\right)$ for a suitable $s \in\{1, \ldots, n\}$. Hence, for some $\gamma \in \mathbb{Z}-\{0\}$, we have $\left(\alpha^{\gamma}\right)^{g^{h}},\left(a^{\gamma}\right)^{g^{k}} \in H_{s}$, and $\left\langle\left(a^{\gamma}\right)^{g^{h}},\left(a^{\gamma}\right)^{g^{k}}\right\rangle$ is abelian. Thus $\left\langle a^{\gamma},\left(a^{\gamma}\right)^{g^{h-k}}\right\rangle$ is abelian, so that there exists $j \epsilon$ $\{1, \ldots, n\}$ for which $\left\langle a^{\gamma},\left(a^{\gamma}\right)^{g^{h-k}}\right\rangle \subseteq I_{G}\left(H_{j}\right)$. Obviously $j=i$, since $a^{\gamma} \in I_{G}\left(H_{i}\right)$ and $H_{i} \cap H_{j}=1$ for $i \neq j$, by (I). Then $a^{g^{h-k}} \in I_{G}\left(H_{i}\right)$ and obviously $a^{g^{h-k}} \notin \bigcup_{j \neq i} I_{G}\left(H_{j}\right)$. For any $a_{1} \in H_{i},\left\langle a^{g^{h-k}}, a_{1}^{g^{h-k}}\right\rangle$ abelian it follows, arguing as before, $\left\langle a^{g^{h-k}}, a_{1}^{g^{h-k}}\right\rangle \subseteq I_{G}\left(H_{i}\right)$; hence the group $H_{i} /\left(H_{i} \cap H_{i}^{g^{k-h}}\right)$ is periodic.

Write $X=\left\langle H_{i}, H_{i}^{g^{k-h}}\right\rangle$, then $H_{i} \cap H_{i}^{g^{k-h}} \triangleleft X$ and writing $\bar{X}=X /\left(H_{i} \cap H_{i}^{g^{k-h}}\right)$, we have $\bar{X} \subseteq \bigcup_{j \neq i} I_{\bar{X}}\left(\overline{H_{j} \cap \bar{X}}\right)$. It follows by induction that $X \subseteq I_{X}\left(\left(H_{j} \cap X\right)\left(H_{i} \cap H_{i}^{g^{k-h}}\right)\right)$ for some $j \in\{1, \ldots, n\}$. From $\left(H_{j} \cap X\right)\left(H_{i} \cap H_{i}^{g^{k-h}}\right)$ soluble it follows, by Theorem A, $\left(H_{i} \cap X\right)\left(H_{i} \cap H_{i}^{g^{k-h}}\right) \sim H_{t} \cap\left(H_{j} \cap X\right)\left(H_{i} \cap H_{i}^{g^{k-h}}\right)$ for some $t$, hence $X \sim H_{t} \cap X$. Obviously the only possibility is $t=i$ and (II) holds.

Now take $a \in H_{1}-\bigcup_{i \neq 1} I_{G}\left(H_{i}\right), b \in H_{2}-\bigcup_{i \neq 2} I_{G}\left(H_{i}\right)$. Then, by (II), there is $\alpha \in \mathbb{Z}-\{0\}$ such that $\left\langle H_{1}, H_{1}^{b^{\alpha}}\right\rangle \subseteq I_{G}\left(H_{1}\right)$, so that, for some $r \in \mathbb{Z}-\{0\},\left[a^{r}, b^{\alpha}\right] \in H_{1}$ and, for every $s \in \mathbb{Z},\left[a^{r}, b^{\alpha}\right]^{s}=\left[a^{r s}, b^{\alpha}\right] \in H_{1}$. Also, by (II), there exists $k \in \mathbb{Z}-\{0\}$ such that $\left\langle H_{2}, H_{2}^{\alpha^{r k}}\right\rangle \subseteq I_{G}\left(H_{2}\right)$, hence $\left[a^{r k}, b^{\alpha}\right] \in I_{G}\left(H_{2}\right)$ and, for some $s \in \mathbb{Z}-\{0\},\left[\alpha^{r k}, b^{\alpha}\right]^{s} \in H_{2}$. 
Thus $\left[a^{r k s}, b^{\alpha}\right]=\left[a^{r k}, b^{\alpha}\right]^{s}=\left[a^{r}, b^{\alpha}\right]^{k s} \in H_{1} \cap H_{2}=1$, and $\left\langle a^{r k s}, b^{\alpha}\right\rangle$ is abelian. Then $\left\langle a^{r k s}, b^{\alpha}\right\rangle \subseteq I_{G}\left(H_{s}\right)$, for some $s \in\{1, \ldots, n\}$; from $a \in I_{G}\left(H_{s}\right)$ it follows $s=1$ and from $b \in I_{G}\left(H_{s}\right), s=2$, the final contradiction.

In order to prove Theorem $\mathrm{C}$, we need the following easy Lemma:

Lemma 3.1. Let $G$ be a group, $G=\bigcup_{i=1}^{n} I_{G}\left(H_{i}\right)$, where $H_{i} \leq G, i=1, \ldots, n$. Assume $G=H_{j} \times H$, for some $j$ and some $H \leq G$. Then either $G=\bigcup_{i \neq j} I_{G}\left(H_{i}\right)$, or $G=I_{G}\left(H_{j}\right)$.

Proof. If $G \neq \bigcup_{i \neq j} I_{G}\left(H_{i}\right)$, there exists $b \in H_{j}, b \notin \bigcup_{i \neq j} I_{G}\left(H_{i}\right)$. For any $a \in H$, consider the elements $a^{m} b, m \in \mathbb{N}$. Then there exists $s \in\{1, \ldots, n\}$ such that $a^{h} b, a^{k} b \in I_{G}\left(H_{s}\right)$ for $h, k \in \mathbb{N}, h \neq k$. Then $\left(a^{h} b\right)^{\beta}=a^{h \beta} b^{\beta} \in H_{s}$ and $\left(a^{k} b\right)^{\beta}=a^{k \beta} b^{\beta} \in H_{s}$, for a suitable $\beta \in \mathbb{N}$ and $a^{\beta(h-k)} \in H_{s}$. Thus $a \in I_{G}\left(H_{s}\right)$ and $b \in I_{G}\left(H_{s}\right)$, since $a^{h} b \in I_{G}\left(H_{s}\right)$, then $s=j$ and $G=I_{G}\left(H_{j}\right)$, as required.

THEOREM C. Let $G$ be a group, $H_{1}, \ldots, H_{n}$ normal subgroups of $G$ such that $G=\bigcup_{i=1}^{n} I_{G}\left(H_{i}\right)$.

Then $G=I_{G}\left(H_{j}\right)$ for some $j \in\{1, \ldots, n\}$.

Proof. By induction on $n$ we may assume $G / H_{1} \subseteq I_{G / H_{1}}\left(H_{j} H_{1} / H_{1}\right)$ for some $j$.

Let $l \geq 1$ be maximum such that

$$
G /\left(H_{1} \cap \ldots \cap H_{l}\right) \sim\left(H_{t}\left(H_{1} \cap \ldots \cap H_{l}\right)\right) /\left(H_{1} \cap \ldots \cap H_{l}\right),
$$

for some $t \in\{1, \ldots, n\}$.

If $l=n$, then the result follows. Assume for a contradiction $l<n$. Without loss of generality we can assume $t=l+1$, so that $G /\left(H_{1} \cap \ldots \cap H_{l}\right) \sim\left(H_{l+1}\left(H_{1} \cap \ldots \cap\right.\right.$ $\left.\left.H_{l}\right)\right) /\left(H_{1} \cap \ldots \cap H_{l}\right)$. Write $X=H_{l+1}\left(H_{1} \cap \ldots \cap H_{l}\right)$, then $X /\left(H_{1} \cap \ldots \cap H_{l+1}\right)=$ $H_{l+1} /\left(H_{1} \cap \ldots \cap H_{l+1}\right) \times\left(H_{1} \cap \ldots \cap H_{l}\right) /\left(H_{1} \cap \ldots \cap H_{l+1}\right)$, by Lemma 3.1 and by induction, we have $X /\left(H_{1} \cap \ldots \cap H_{l+1}\right) \sim\left(\left(H_{s} \cap X\right)\left(H_{1} \cap \ldots \cap H_{l+1}\right)\right) /\left(H_{1} \cap \ldots \cap H_{l+1}\right)$ for some $s \in\{1, \ldots, n\}$. Thus $G /\left(H_{1} \cap \ldots \cap H_{l+1}\right) \sim\left(H_{s}\left(H_{1} \cap \ldots \cap H_{l+1}\right)\right) /\left(H_{1} \cap \ldots \cap H_{l+1}\right)$ because $G \sim X$, contradicting the maximality of $l$.

Corollary 3.2(†). Let $G$ be a group, $H_{1}, \ldots, H_{n}$ subnormal subgroups of $G$ such that $G=\bigcup_{i=1}^{n} I_{G}\left(H_{i}\right)$. Then $G=I_{G}\left(H_{j}\right)$ for some $j \in\{1, \ldots, n\}$.

Proof. Denote by $m_{i}$ the subnormal defect of $H_{i}$, for any $i \in\{1, \ldots, n\}$. We argue by induction on the sum of the $m_{i}$ 's. By Theorem $\mathrm{C}, G=I_{G}\left(H_{j}^{G}\right)$ for some $j$. But $H_{j} \triangleleft^{m_{j}-1} H_{j}^{G}$, and $H_{i} \cap H_{j}^{G} \triangleleft^{m_{i}} H_{j}^{G}$ for $i \neq j$. So $H_{j}^{G}=I_{H_{j}^{G}}\left(H_{i} \cap H_{j}^{G}\right)$ for some $i$ and $G=I_{G}\left(H_{i}\right)$, as required.

\section{REFERENCES}

1. M. A. Brodie, R. F. Chamberlain and L.-C. Kappe, Finite coverings by normal subgroups, Proc. Amer. Math. Soc., 104 no. 3 (1988), 669-674.

† The authors wish to thank Professor Howard Smith for this remark. 
2. L.-C. Kappe, Finite coverings by 2-Engel groups, Bull. Austral. Math. Soc., 38 (1988), $141-150$.

3. A. S. Kirkinskii, Intersection of finitely generated subgroups in metabelian groups, Algebra and Logic, 20 no. 1 (1981), 22-36 (Algebra i Logika, 37-54).

4. J. C. Lennox, Bigenetic properties of finitely generated hyper-(abelian-by-finite) groups, $J$. Austral. Math. Soc., 16 (1973), 309-315.

5. J. C. Lennox and J. Wiegold, Extension of a problem of Paul Erdös on groups, J. Austral. Math. Soc., Ser. A, 31 (1981), 459-463.

6. B. H. Neumann, Groups covered by permutable subsets, J. London Math. Soc., 29 (1954), $236-248$.

7. A. H. Rhemtulla and B. A. F. Wehrfritz, Isolators in soluble groups of finite rank, Rocky Mountain J. Math., 14 no. 2 (1984), 415-421.

8. D. J. S. Robinson, Finiteness conditions and generalized soluble groups, vol. I, II, Springer-Verlag, Berlin-New York, 1972.

9. M. J. Tomkinson, Hypercentre-by-finite groups, Publ. Math. Debrecen 40 (1992), 313-321.

P. Longobardi and M. Maj

Dipartimento di Matematica e Applicazioni

Universita' degli StUdi di NAPOLI

via Cinthia, Monte S. Angelo

80126 NAPLES-ITALY
A. H. Rhemtulla
DEPT. OF MATHEMATICS
University of Alberta
Edmonton, Alberta
Canada T6G 2G1 